\title{
Reducing Electron Dose and Sample Damage with Bayesian Machine Learning and Self-Organizing Neural Networks
}

\author{
Karl Hujsak ${ }^{1}$, Benjamin D. Myers ${ }^{1,2}$, Eric Roth ${ }^{1,2}$, Yue Li $^{3}$, Vinayak P. Dravid ${ }^{1,2,3}$
}

1. Department of Materials Science \& Engineering, Northwestern University, Evanston, Illinois 602083108

2. Electron Probe and Instrumentation Center, NUANCE, Northwestern University, Evanston, Illinois 60208-3108

3. Applied Physics Program, Northwestern University, Evanston, Illinois 60208-3108

There is a pressing need in the electron microscopy community to identify new methods to strongly reduce the sensitivity of observed specimens to the strong electron currents modern microscopes are capable of. For many nontraditional materials, such as biological or hybrid polymeric structures, the limiting factor is rarely the power of the actual microscope, but instead the sample's structural integrity to the intense and finely focused electron probe. These specimens are particularly sensitive to effects of radiolysis and electrostatic charging, which often prevent more than a one-shot capture of the area of interest $^{1}$. New studies involving in-situ fluidic or gaseous environments are also suffering from significant electron medium interactions, which can often preclude quantitative measurements in such environments ${ }^{2}$. These studies are complicated by the need to capture dynamic phenomena with multiple exposures, which can significantly increase the number of electrons injected per unit area.

Previous approaches to limit sample specimen interactions have involved reducing exposures, which result in noisy and poor quality images. For many samples there may not be an acceptable exposure time that precludes sample damage while ensuring a sufficient signal-to-noise-ratio, particularly if multiple images are needed to fully capture a dynamic or transient phenomena.

In this presentation an alternative approach based on statistical redundancies within most electron images is exploited to drastically reduce the relative electron exposure per image (often by up to 70$80 \%$ ) while preserving image quality. We propose the novel use of an electrostatic beam blanker to act as an electrostatic shutter in which pixels can programmatically be rejected or sampled by the electron beam. The sampled pixels are collected into image patches and the underlying statistical structure is explored utilizing the Beta Process Factor Analysis Algorithm (BPFA).

The BPFA algorithm is a type of dictionary learning algorithm, in which the image is decomposed into a large dictionary which stores common features from the sparsely observed pixels and an activation matrix, in which dictionary features are linearly combined to recover the full image ${ }^{3}$. This algorithm has been shown to achieve high quality statistical reconstruction from simulated sparse electron images previously, and requires no pre-training of the dictionary, forcing the algorithm to recover the full image only from the few observed pixels ${ }^{4}$. This encourages generality, while still allowing reconstructions to be performed at extraordinarily low sampling percentages.

The use of an electrostatic beam blanker as opposed to a mechanical shutter or direct beam control allows for easy implementation in many research facilities, due to the prevalence of beam blankers in 
electron beam lithography. Thus, the transition between full sampling and random-undersampling can be quickly done on the fly, allowing a user to fully align the microscope on a nearby area and easily transition to sparse imaging during the final exposure of the feature of interest. Less computational intensive in-painting approaches can then quickly be used to confirm the presence of such a feature, and a full statistical reconstruction performed offline. In this way, sample damage can be traded for afterhours processing time, a small price to pay for previously inaccessibly low area doses with high image quality. The use of random sampling masks further allows for multiple sparse images to be captured at the cost of one full image, allowing five $20 \%$ sampled images to be captured in which no pixel is sampled more than once.

Suppression of real dose related artifacts in a variety of material, biological, and in-situ fluidic samples are demonstrated in a commercial scanning electron microscope (SEM). Limits and effective practices are discussed and examined in the context of dose limited microscopy, as well as potential new avenues for Bayesian learning in electron and scan probe microscopy.

References:

[1] GLAESER, R.M. \& TAYLOR, K.A. (1978). Radiation damage relative to transmission electron microscopy of biological specimens at low temperature: a review. Journal of Microscopy 112(1), 127-138.

[2] Woehl, T.J., Jungjohann, K.L., Evans, J.E., Arslan, I., RistenPart, W.D. \& Browning, N.D. (2013). Experimental procedures to mitigate electron beam induced artifacts during in situ fluid imaging of nanomaterials. Ultramicroscopy 127, 53-63.

[3] Zhou, M., Chen, H., Paisley, J., Ren, L., Li, L., Xing, Z., Dunson, D., SAPIRO, G. \& Carin, L. (2012). Nonparametric Bayesian dictionary learning for analysis of noisy and incomplete images. Image Processing, IEEE Transactions on 21(1), 130-144.

[4] Stevens, A., YAng, H., CARIN, L., Arslan, I. \& Browning, N.D. (2014). The potential for Bayesian compressive sensing to significantly reduce electron dose in high-resolution STEM images. Microscopy 63(1), 41-51.

[5] This work is based upon work supported by the Air Force Office of Scientific Research under Award No. FA9550-12-1-0280 and the Air Force Research Laboratory under Award No. FA8650-15-2-5518.

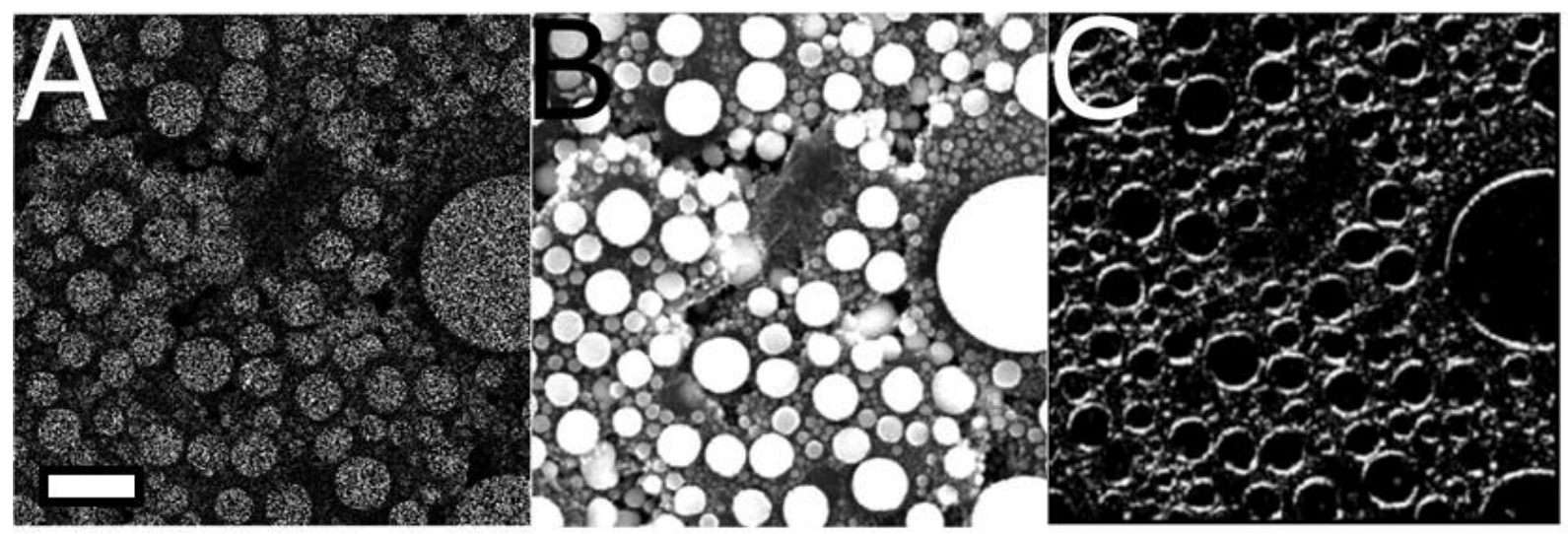

Figure 1. (a) Randomly under-sampled image with $30 \%$ observed pixels and (b) the statistical reconstruction (scale bar $=300 \mathrm{~nm}$ ). (c) Segmented edges of the sparse image utilizing learned dictionary features and a self-organizing neural network. 